\title{
A Note on Double Laplace Transform and Telegraphic Equations
}

\author{
Hassan Eltayeb ${ }^{1}$ and Adem Kiliçman ${ }^{2}$ \\ ${ }^{1}$ Department of Mathematics, College of Science, King Saud University, P.O. Box 2455, Riyadh 11451, Saudi Arabia \\ ${ }^{2}$ Department of Mathematics and Institute for Mathematical Research, Universiti Putra Malaysia, 43400 Serdang, \\ Selangor, Malaysia
}

Correspondence should be addressed to Adem Kiliçman; akilicman@putra.upm.edu.my

Received 12 November 2012; Accepted 2 January 2013

Academic Editor: Mustafa Bayram

Copyright (C) 2013 H. Eltayeb and A. Kiliçman. This is an open access article distributed under the Creative Commons Attribution License, which permits unrestricted use, distribution, and reproduction in any medium, provided the original work is properly cited.

Double Laplace transform is applied to solve general linear telegraph and partial integrodifferential equations. The scheme is tested through some examples, and the results demonstrate reliability and efficiency of the proposed method.

\section{Introduction}

The wave equation is known as one of three fundamental equations in mathematical physics and occurs in many branches of physics, applied mathematics, and engineering. It is also known that there are two types of these equation: the homogenous equation that has constant coefficient with many classical solutions such as separation of variables [1], the methods of characteristics $[2,3]$, single Laplace transform, and Fourier transform [4] and nonhomogenous equations with constant coefficient solved by double Laplace transform [5] and operation calculus [6].

In this study, we use double Laplace transform to solve telegraph equation and partial integrodifferential equation. We follow the method that was proposed by Kilıçman and Eltayeb [7] where they extended one-dimensional convolution theorem to two-dimensional case [8].

First of all, we recall the following definitions given by Kılıçman and Gadain [9]. The double Laplace transform is defined by

$$
L_{x} L_{t}[f(x, s)]=F(p, s)=\int_{0}^{\infty} e^{-p x} \int_{0}^{\infty} e^{-s t} f(x, t) d t d x,
$$

where $x, t>0$ and $p, s$ are complex numbers,

and the first-order partial derivative is defined as follows:

$$
L_{x} L_{t}\left[\frac{\partial f(x, t)}{\partial x}\right]=p F(p, s)-F(0, s) .
$$

Double Laplace transform for second partial derivative with respect to $x$ is given by

$$
L_{x x}\left[\frac{\partial^{2} f(x, t)}{\partial^{2} x}\right]=p^{2} F(p, s)-p F(0, s)-\frac{\partial F(0, s)}{\partial x},
$$

and double Laplace transform for second partial derivative with respect to $t$ similarly as the previous is given by

$$
L_{t t}\left[\frac{\partial^{2} f(x, t)}{\partial^{2} t}\right]=s^{2} F(p, s)-s F(p, 0)-\frac{\partial F(p, 0)}{\partial t} .
$$

In a similar manner, the double Laplace transform of a mixed partial derivative can be deduced from a single Laplace transform as

$$
\begin{aligned}
L_{x} L_{t} & {\left[\frac{\partial^{2} f(x, t)}{\partial x \partial t}\right] } \\
& =p s F(p, s)-p F(p, 0)-s F(0, s)-F(0,0) .
\end{aligned}
$$

Theorem 1. If at the point $(p, q)$ the integral

$$
F_{1}(p, q)=\int_{0}^{\infty} \int_{0}^{\infty} e^{-p x-q y} f_{1}(x, y) d x d y
$$

is convergent and the integral

$$
F_{2}(p, q)=\int_{0}^{\infty} \int_{0}^{\infty} e^{-p x-q y} f_{2}(x, y) d x d y
$$


is absolutely convergent, then

$$
F(p, q)=F_{1}(p, q) F_{2}(p, q)
$$

is the Laplace transform of the function

$$
f(x, y)=\int_{0}^{x} \int_{0}^{y} f_{1}(x-\zeta, y-\eta) f_{2}(\zeta, \eta) d \zeta d \eta
$$

and the integral

$$
F(p, q)=\int_{0}^{\infty} \int_{0}^{\infty} e^{-p x-q y} f(x, y) d x d y
$$

is convergent at the point $(p, q)$.

Proof. See [4].

Next, we study the uniqueness and existences of double Laplace transform. First of all, let $f(x, t)$ be a continuous function on the interval $[0, \infty)$ which is of exponential order, that is, for some $a, b \in \mathbb{R}$. Consider

$$
\sup _{\substack{t>0 \\ x>0}} \frac{|f(x, t)|}{e^{a x+b t}}<\infty
$$

In this case, the double Laplace transform of

$$
F(p, s)=\int_{0}^{\infty} \int_{0}^{\infty} e^{-s t-p x} f(x, t) d x d t
$$

exists for all $p>a$ and $s>b$ and is in fact infinitely differentiable with respect to $p>a$ and $s>b$. All functions in this study are assumed to be of exponential order. The following theorem shows that $f(x, t)$ can be uniquely recovered from $F(p, s)$.

Theorem 2. Let $f(x, t)$ and $g(x, t)$ be continuous functions defined for $x, t \geq 0$ and having Laplace transforms, $F(p, s)$, and $G(p, s)$, respectively. If $F(p, s)=G(p, s)$, then $f(x, t)=g(x, t)$.

Proof. If $\alpha$ and $\beta$ are sufficiently large, then the integral representation, by

$$
f(x, t)=\frac{1}{2 \pi i} \int_{\alpha-i \infty}^{\alpha+i \infty} e^{p x}\left(\frac{1}{2 \pi i} \int_{\beta-i \infty}^{\beta+i \infty} e^{s t} F(p, s) d s\right) d p
$$

for the double inverse Laplace transform, can be used to obtain

$$
\begin{aligned}
f(x, t) & =\frac{1}{2 \pi i} \int_{\alpha-i \infty}^{\alpha+i \infty} e^{p x}\left(\frac{1}{2 \pi i} \int_{\beta-i \infty}^{\beta+i \infty} e^{s t} F(p, s) d s\right) d p \\
& =\frac{1}{2 \pi i} \int_{\alpha-i \infty}^{\alpha+i \infty} e^{p x}\left(\frac{1}{2 \pi i} \int_{\beta-i \infty}^{\beta+i \infty} e^{s t} G(p, s) d s\right) d p \\
& =g(x, t),
\end{aligned}
$$

Theorem 3. A function $f(x, t)$ which is continuous on $[0, \infty)$ and satisfies the growth condition (11) can be recovered from $F(p, s)$ as

$$
f(x, t)=\lim _{\substack{m \rightarrow \infty \\ n \rightarrow \infty}} \frac{(-1)^{m+n}}{m ! n !}\left(\frac{m}{x}\right)^{m+1}\left(\frac{n}{t}\right)^{n+1} \Psi^{m+n}\left(\frac{m}{x}, \frac{n}{t}\right),
$$

where $\Psi^{m+n}$ is denoted by $(m+n)$ th mixed partial derivatives of $F(p, s)$, defined by $\Psi^{m+n}=\partial^{m+n} F(p, s) / \partial p^{m} \partial s^{n}$ for $x, t \geq 0$ since the previous theorem obtains $f(x, t)$ in terms of $F(p, s)$.

Of course, the main difficulty in using Theorem 3 for computing the inverse Laplace transform is the repeated symbolic differentiation of $F(p, s)$. However, one can apply Theorem 3 in the next type of examples.

Example 4. Let $f(x, t)=e^{-a x-b t}$. The Laplace transform is easily found to be as follows:

$$
F(p, s)=\frac{1}{(p+a)(s+b)}
$$

It is also simple to verify that

$$
\frac{\partial^{m+n} F(p, s)}{\partial p^{m} \partial s^{n}}=m ! n !(-1)^{m+n}(p+a)^{-m-1}(s+b)^{-n-1} .
$$

Putting this expression for $\partial^{m+n} F(p, s) / \partial p^{m} \partial s^{n}$ into Theorem 3 gives the following:

$$
\begin{aligned}
f(x, t) & =\lim _{\substack{m \rightarrow \infty \\
n \rightarrow \infty}} \frac{m^{m+1} n^{n+1}}{x^{m+1} t^{n+1}}\left(a+\frac{m}{x}\right)^{-m-1}\left(b+\frac{n}{t}\right)^{-n-1} \\
& =\lim _{\substack{m \rightarrow \infty \\
n \rightarrow \infty}}\left(1+\frac{a x}{m}\right)^{-m-1}\left(1+\frac{b t}{n}\right)^{-n-1} .
\end{aligned}
$$

The last limit is easy to evaluate. Take the natural log of both sides, and write the result in the form of $-(\ln (1+$ $a x / m) /(1 /(m+1)))-(\ln (1+b t / n) /(1 /(n+1)))$. L'Hopital's rule reveals that the indeterminate from approaches $-a x-b t$. The continuity of the natural logarithm shows that $\ln (f(x, t))=$ $-a x-b t$; then, $f(x, t)=e^{-a x-b t}$.

\section{Properties of Double Laplace Transform}

In this part, we consider some of the properties of the double Laplace Transform that will enable us to find further transform pairs $\{f(x, t), F(p, s)\}$ without having to compute consider the following.

(I) $F(p+d, s+c)=L_{x} L_{t}\left[e^{-d x-c t} f(x, t)\right](p, s)$,
(II) $\frac{1}{k} F\left(\frac{p}{\alpha}, \frac{s}{\beta}\right)=L_{x} L_{t}[f(\alpha x, \beta t)](p, s)$,

where $k=\alpha \beta$,

(III) $\frac{\partial^{m+n} F(p, s)}{\partial p^{m} \partial^{n}}=L_{x} L_{t}\left[(-1)^{m+n} x^{m} t^{n} f(x, t)\right](p, s)$.

and the theorem is proven. 
Was first verify (I) as

$$
\begin{aligned}
L_{x} L_{t} & {\left[e^{-d x-c t} f(x, t)\right](p, s) } \\
& =\int_{0}^{\infty} \int_{0}^{\infty} e^{-s t-p x} e^{-d x-c t} f(x, t) d t d x \\
& =\int_{0}^{\infty} e^{-d x-p x}\left(\int_{0}^{\infty} e^{-s t-c t} f(x, t) d t\right) d x .
\end{aligned}
$$

We calculate the integral inside bracket as

$$
\int_{0}^{\infty} e^{-s t-c t} f(x, t) d t=F(x, s+c) .
$$

By substituting, we obtain

$$
\begin{aligned}
L_{x} L_{t}\left[e^{-d x-c t} f(x, t)\right](p, s) & =\int_{0}^{\infty} e^{-d x-p x} F(x, s+c) d x \\
& =F(p+d, s+c) .
\end{aligned}
$$

Second, the right hand side of (II) can be written in the form of

$$
\begin{aligned}
L_{x} L_{t}[ & f(\alpha x, \beta t)](p, s) \\
& =\int_{0}^{\infty} \int_{0}^{\infty} e^{-s t-p x} f(\alpha x, \beta t) d x d t \\
& =\int_{0}^{\infty} e^{-s t}\left(\int_{0}^{\infty} e^{-p x} f(\alpha x, \beta t) d x\right) d t \\
& =\int_{0}^{\infty} e^{-s t} \frac{1}{\alpha} F\left(\frac{p}{\alpha}, \beta t\right) d t=\frac{1}{\alpha \beta} F\left(\frac{p}{\alpha}, \frac{s}{\beta}\right) .
\end{aligned}
$$

The last property, from definition of double Laplace transform

$$
F(p, s)=\int_{0}^{\infty} \int_{0}^{\infty} e^{-s t-p x} f(x, t) d x d t
$$

so that

$$
\frac{\partial^{m+n} F(p, s)}{\partial p^{m} \partial s^{n}}=\frac{\partial^{m+n}}{\partial p^{m} \partial s^{n}} \int_{0}^{\infty} \int_{0}^{\infty} e^{-s t-p x} f(x, t) d x d t
$$

Owing to the convergence properties of the improper integral involved, we can interchange the operation of differentiation and integration and differentiate with respect to $p$, $s$ under the integral sign. Thus,

$$
\frac{\partial^{m+n} F(p, s)}{\partial p^{m} \partial s^{n}}=\int_{0}^{\infty} \frac{\partial^{n}}{\partial s^{n}} e^{-s t}\left(\int_{0}^{\infty} \frac{\partial^{m}}{\partial p^{m}} e^{-p x} f(x, t) d x\right) d t
$$

which, on carrying out the repeated differentiation with respect to $p, s$, gives the following:

$$
\begin{aligned}
\frac{\partial^{m+n} F(p, s)}{\partial p^{m} \partial s^{n}} & =(-1)^{m+n} \int_{0}^{\infty} \int_{0}^{\infty} x^{m} t^{n} e^{-s t-p x} f(x, t) d x d t \\
& =(-1)^{m+n} L_{x} L_{t}\left[x^{m} t^{n} f(x, t)\right](p, s) .
\end{aligned}
$$

The previous three properties are very useful at the proof of Theorem 3.

Proof of Theorem 3. Let us define the set of functions depending on parameters $m, n$ as

$$
\begin{gathered}
g_{m, n}(x, t)=\frac{m^{m+1} n^{n+1}}{m ! n !} x^{m} t^{n} e^{-m x-n t} \\
\text { so } \int_{0}^{\infty} \int_{0}^{\infty} g_{m, n}(x, t) d x d t=1, \\
\lim _{m \rightarrow \infty} \lim _{n \rightarrow \infty} \int_{0}^{\infty} \int_{0}^{\infty} g_{m, n}(x, t) \varphi(x, t) d x d t=\varphi(1,1),
\end{gathered}
$$

where $\varphi(x, t)$ is any continuous function. Let us denote its Laplace transform as a function of $p, s$ by $L_{x} L_{t}[\varphi(x, t)](p, s)$. Now, we define the function $\Psi(x, t)=f\left(x x_{0}, t t_{0}\right)$, and using property (II), we have

$$
\begin{aligned}
& L_{x} L_{t} {[\Psi(x, t)](p, s) } \\
&=L_{x} L_{t}\left[f\left(x x_{0}, t t_{0}\right)\right](p, s)=\frac{1}{x_{0} t_{0}} F\left(\frac{p}{x_{0}}, \frac{s}{t_{0}}\right) .
\end{aligned}
$$

We apply property (III) (we must evaluate the $m+n$ mixed partial derivatives of $F(p, s)$ at the points $p=m / x$ and $s=$ $n / t)$ as follows:

$$
\begin{aligned}
& \frac{\partial^{m+n}}{\partial p^{m} \partial s^{n}}\left(L_{x} L_{t}[\Psi(x, t)]\right)(p, s) \\
& \quad=\frac{1}{x_{0}^{m+1} t_{0}^{n+1}} \frac{\partial^{m+n}}{\partial p^{m} \partial s^{n}} F\left(\frac{p}{x_{0}}, \frac{s}{t_{0}}\right) .
\end{aligned}
$$

Let $\varphi(x, t)=e^{-p x-s t} \Psi(x, t)$. By using (28), we have

$$
\begin{aligned}
\varphi(1,1)= & e^{-p-s} \Psi(1,1)=e^{-p-s} f\left(x_{0}, t_{0}\right) \\
= & \lim _{m \rightarrow \infty} \lim _{n \rightarrow \infty} \frac{m^{m+1} n^{n+1}}{m ! n !} \\
& \times \int_{0}^{\infty} \int_{0}^{\infty} x^{m} t^{n} e^{-p x-s t} e^{-m x-n t} \Psi(x, t) d x d t \\
= & \lim _{m \rightarrow \infty} \lim _{n \rightarrow \infty} \frac{m^{m+1} n^{n+1}}{m ! n !} \\
& \times L_{x} L_{t}\left[x^{m} t^{n} e^{-m x-n t} \Psi(x, t)\right](p, s) .
\end{aligned}
$$

By using the previous properties (I) and (II) of double Laplace transform, (30), and the definition of $\Psi(x, t)$, we have

$$
\begin{aligned}
& L_{x} L_{t}\left[x^{m} t^{n} e^{-m x-n t} \Psi(x, t)\right](p, s) \\
& \quad=(-1)^{m+n} \frac{\partial^{m+n}}{\partial p^{m} \partial s^{n}}\left(L_{x} L_{t}\left(e^{-m x-n t} \Psi(x, t)\right)\right)(p, s) \\
& \quad=(-1)^{m+n} \frac{\partial^{m+n}}{\partial p^{m} \partial s^{n}}\left(L_{x} L_{t}(\Psi(x, t))\right)(p+m, s+n) \\
& =(-1)^{m+n} \frac{1}{z} \frac{\partial^{m+n}}{\partial p^{m} \partial s^{n}}\left(L_{x} L_{t}\left(f\left(x x_{0}, t t_{0}\right)\right)\right)\left(\frac{p+m}{x_{0}}, \frac{s+n}{t_{0}}\right) \\
& =(-1)^{m+n} \frac{1}{z} \frac{\partial^{m+n}}{\partial p^{m} \partial s^{n}}\left(F\left(\frac{p+m}{x_{0}}, \frac{s+n}{t_{0}}\right)\right),
\end{aligned}
$$


where $1 / z=1 / x_{0}^{m+1} t_{0}^{n+1}$. From (31) and (32), with $f\left(x_{0}, t_{0}\right)=$ $e^{p+s} \varphi(1,1)$, we have

$$
\begin{gathered}
f\left(x_{0}, t_{0}\right) \\
=e^{p+s} \lim _{m \rightarrow \infty} \lim _{n \rightarrow \infty} \frac{(-1)^{m+n}}{m ! n !}\left(\frac{m}{x_{0}}\right)^{m+1}\left(\frac{n}{t_{0}}\right)^{n+1} \\
\times \frac{\partial^{m+n}}{\partial p^{m} \partial s^{n}}\left(F\left(\frac{p+m}{x_{0}}, \frac{s+n}{t_{0}}\right)\right) .
\end{gathered}
$$

For any $p, s$ the statement in Theorem 3 is actually just the special case for $p=0$ and $s=0$.

Example 5. Find double Laplace transform for a regular generalized function

$$
\begin{gathered}
f(x, t)=H(t) \otimes H(x) \ln (t) \ln (x), \\
\frac{\partial^{2}}{\partial x \partial t} f(x, t)=\frac{\partial^{2}}{\partial x \partial t}[H(t) \otimes H(x) \ln (t) \ln (x)] \\
=p f\left[\frac{H(t) \otimes H(x)}{x t}\right],
\end{gathered}
$$

where $H(x, t)=H(t) \otimes H(x)$ is a Heaviside function, and $\otimes$ is tensor product. The double Laplace transform with respect to $x, t$ of (1) becomes

$$
\begin{gathered}
L_{x} L_{t}[f(x, t)]=\int_{0}^{\infty} e^{-p x} \ln (x) \int_{0}^{\infty} e^{-s t} \ln (t) d t d x \\
=-\frac{1}{s} \int_{0}^{\infty} e^{-p x} \ln (x)[\gamma+\ln s] d x, \\
L_{x} L_{t}[f(x, t)]=\frac{1}{s p}\left[\gamma^{2}+\ln (p) \ln (s)\right],
\end{gathered}
$$

where $\gamma$ is Euler's constant [10]. Thus,

$$
L_{x} L_{t}[f(x, t)]=\frac{1}{s p}\left[\gamma^{2}+\ln (p) \ln (s)\right], \quad \text { where } R e>0 .
$$

Double Laplace transform of (35) with respect to $x$ and $t$ is obtained as follows:

$$
\begin{aligned}
L_{x} L_{t}\left[\frac{\partial^{2}}{\partial x \partial t} f(x, t)\right] & =L_{x} L_{t}[H(t) H(x) \ln (t) \ln (x)] \\
& =p s\left[\frac{1}{s p}\left[\gamma^{2}+\ln (p) \ln (s)\right]\right] \\
& =\gamma^{2}+\ln (p) \ln (s) .
\end{aligned}
$$

Definition 6. A linear continuous function over the space $L$ of test functions is called a distribution of exponential growth. This dual space of $L$ is denoted by $L^{\prime}[10]$.

Example 7. Let us find double laplace transform of the function $\left(x^{\alpha}+t^{\beta}\right)=H(x) \otimes H(t) x^{\alpha} t^{\beta}$, where $\alpha, \beta \neq-1,-2, \ldots$
Since $\left(x^{\alpha}+t^{\beta}\right) \in L^{\prime}$, then double laplace transform of the function $\left(x^{\alpha}+t^{\beta}\right)=H(x) \otimes H(t) x^{\alpha} t^{\beta}$ is given by

$$
L_{x} L_{t}\left[\left(x^{\alpha}+t^{\beta}\right)\right]=\int_{0}^{\infty} x^{\alpha} e^{-p x} \int_{0}^{\infty} t^{\beta} e^{-s t} d t d x
$$

Letting $u=p x$ and $v=s t$ for $p, t>0$, it follows that

$$
\begin{aligned}
L_{x} L_{t}\left[\left(x^{\alpha}+t^{\beta}\right)\right] & =\frac{1}{p^{\alpha+1} s^{\beta+1}} \int_{0}^{\infty} u^{\alpha} e^{-u} \int_{0}^{\infty} v^{\beta} e^{-v} d v d u, \\
& =\frac{1}{p^{\alpha+1} s^{\beta+1}} \Gamma(\alpha+1) \Gamma(\beta+1) .
\end{aligned}
$$

In particular, if $\alpha, \beta=0$, (40) becomes

$$
L_{x} L_{t}[H(x) \otimes H(t)]=\int_{0}^{\infty} e^{-p x} \int_{0}^{\infty} e^{-s t} d t d x=\frac{1}{p s} .
$$
form:

Consider the general telegraph equation in the following

$$
u_{x x}=u_{t t}+u_{t}+u+f(x, t)
$$

with boundary conditions

$$
u(0, t)=f_{1}(t), \quad u_{x}(0, t)=f_{2}(t),
$$

and initial conditions

$$
u(x, 0)=g_{1}(x), \quad u_{t}(x, 0)=g_{2}(x) .
$$

We apply double Laplace transform for (42) and single Laplace transform for (43) and (43). after taking double inverse Laplace transform, we obtain the solution of (42) in the form

$$
\begin{aligned}
u(x, t)=L_{p}^{-1} L_{s}^{-1}[ & \frac{F(p, s)+p F_{1}(s)+F_{2}(s)}{\left(p^{2}-s^{2}-s-1\right)} \\
& \left.-\frac{s G_{1}(p)-G_{2}(p)-G_{1}(p)}{\left(p^{2}-s^{2}-s-1\right)}\right] .
\end{aligned}
$$

Here, we assume that the double inverse Laplace transform exists for each term in the right side of (45).

Example 8. Consider the homogeneous telegraph equation given by

$$
u_{x x}-u_{t t}-u_{t}-u=0
$$

with boundary conditions

$$
u(0, t)=e^{-t}, \quad u_{x}(0, t)=e^{-t},
$$

and initial conditions

$$
u(x, 0)=e^{x}, \quad u_{x}(x, 0)=-e^{x} .
$$


Solution 1. By taking double Laplace transform for (46) and single Laplace transform for (47) and (48), we have

$$
\begin{aligned}
U(p, s) & =\frac{\left(p^{2}-s^{2}-s-1\right)}{(s+1)(p-1)\left(p^{2}-s^{2}-s-1\right)} \\
& =\frac{1}{(s+1)(p-1)} .
\end{aligned}
$$

By using double inverse Laplace transform for (49), we get the solution as follows:

$$
u(x, t)=e^{x-t}
$$

In the next example we apply double Laplace transform for nonhomogenous telegraphic equation as follows.

Example 9. Consider the nonhomogenous telegraphic equation denoted by

$$
u_{x x}-u_{t t}-u_{t}-u=-2 e^{x+t}
$$

with boundary conditions

$$
u(0, t)=e^{t}, \quad u_{x}(0, t)=e^{t}
$$

and initial conditions

$$
u(x, 0)=e^{x}, \quad u_{x}(x, 0)=e^{x} .
$$

By taking double Laplace transform for (51) and single Laplace transform for (52) and (53), we have

$$
\begin{aligned}
U(p, s) & =\frac{\left(p^{2}-s^{2}-s-1\right)}{(s-1)(p-1)\left(p^{2}-s^{2}-s-1\right)} \\
& =\frac{1}{(s-1)(p-1)} .
\end{aligned}
$$

By applying double inverse Laplace transform for (54), we get the solution of (51) in the following form:

$$
u(x, t)=e^{x+t}
$$

\section{An Application to Partial Integrodifferential Equations}

Consider the following partial integrodifferential equation:

$$
\begin{gathered}
u_{t t}-u_{x x}+u+\int_{0}^{x} \int_{0}^{t} g(x-\alpha, t-\beta) u(\alpha, \beta) d \alpha d \beta \\
=f(x, t),
\end{gathered}
$$

with boundary conditions

$$
u(0, t)=f_{1}(t), \quad u_{x}(0, t)=f_{2}(t)
$$

and initial conditions

$$
u(x, 0)=g_{1}(x), \quad u_{t}(x, 0)=g_{2}(x) .
$$

By taking double Laplace transform for (56) and single Laplace transform for (57) and (58), we get

$$
\begin{aligned}
U(p, s)= & \frac{p / G_{1}(p)+1 / G_{2}(p)}{\left(p^{2}-s^{2}+1+G(p, s)\right)} \\
& -\frac{p / F_{1}(s)-1 / F_{2}(s)+F(p, s)}{\left(p^{2}-s^{2}+1+G(p, s)\right)} .
\end{aligned}
$$

By applying double inverse Laplace transform for (59), we obtain the solution of (56) in the following form:

$$
\begin{aligned}
u(x, t)=L_{p}^{-1} L_{s}^{-1} & {\left[\frac{p / G_{1}(p)+1 / G_{2}(p)}{\left(p^{2}-s^{2}+1+G(p, s)\right)}\right.} \\
& \left.-\frac{p / F_{1}(s)-1 / F_{2}(s)+F(p, s)}{\left(p^{2}-s^{2}+1+G(p, s)\right)}\right] .
\end{aligned}
$$

We provide the double inverse Laplace transform existing for each terms in the right side of (60). In particular, consider the following example.

Example 10. Consider the partial integro-differential equation

$$
u_{t t}-u_{x x}+u+\int_{0}^{x} \int_{0}^{t} e^{x-\alpha+t-\beta} u(\alpha, \beta) d \alpha d \beta=e^{x+t}+x t e^{x+t}
$$

with conditions

$$
\begin{array}{cc}
u(x, 0)=e^{x}, & u_{t}(x, 0)=e^{x}, \\
u(0, t)=e^{t}, & u_{x}(0, t)=e^{t} .
\end{array}
$$

By taking double Laplace transform for (61) and single Laplace transform for (62), we have

$$
\begin{aligned}
\left(s^{2}-\right. & \left.p^{2}+1+\frac{1}{(p-1)(s-1)}\right) U(p, s) \\
= & \frac{s}{p-1}+\frac{1}{p-1}-\frac{p}{s-1}-\frac{1}{s-1} \\
& +\frac{1}{(p-1)(s-1)}+\frac{1}{(p-1)^{2}(s-1)^{2}} .
\end{aligned}
$$

By simplifying (63), we obtain

$$
U(p, s)=\frac{1}{(p-1)(s-1)} .
$$

By using double inverse Laplace transform for (64), we obtain the solution of (61) as follows:

$$
u(x, t)=e^{x+t} .
$$

\section{Acknowledgments}

The authors would like to express their sincere thanks and gratitude to the reviewers for their valuable comments and suggestions for the improvement of this paper. The first author gratefully acknowledges that this project was partially supported by the Research Center, College of Science, King Saud University. 


\section{References}

[1] G. L. Lamb Jr, Introductory Applications of Partial Differential Equations with Emphasis on Wave Propagation and Diffusion, John Wiley \& Sons, New York, NY, USA, 1995.

[2] U. T. Myint, Partial Differential Equations of Mathematical Physics, American Elsevier, New York, NY, USA, 1980.

[3] C. Constanda, Solution Techniques for Elementary Partial Differential Equations, Chapman \& Hall/CRC, New York, NY, USA, 2002.

[4] D. G. Duffy, Transform Methods for Solving Partial Differential Equations, CRC Press, New York, NY, USA, 2004.

[5] A. Babakhani and R. S. Dahiya, "Systems of multi-dimensional Laplace transforms and a heat equation," in Proceedings of the 16th Conference on Applied Mathematics, vol. 7 of Electronic Journal of Differential Equations, pp. 25-36, University of Central Oklahoma, Edmond, Okla, USA, 2001.

[6] Y. A. Brychkov, H.-J. Glaeske, A. P. Prudnikov, and V. K. Tuan, Multidimensional Integral Transformations, Gordon and Breach Science Publishers, Philadelphia, Pa, USA, 1992.

[7] A. Kılıçman and H. Eltayeb, "A note on the classifications of hyperbolic and elliptic equations with polynomial coefficients," Applied Mathematics Letters, vol. 21, no. 11, pp. 1124-1128, 2008.

[8] H. Eltayeb, A. Kılıçman, and P. Ravi Agarwal, "An analysis on classifications of hyperbolic and elliptic PDEs," Mathematical Sciences, vol. 6, p. 47, 2012.

[9] A. Kılıçman and H. E. Gadain, "On the applications of Laplace and Sumudu transforms," Journal of the Franklin Institute, vol. 347, no. 5, pp. 848-862, 2010.

[10] R. P. Kanwal, Generalized Functions Theory and Applications, Birkhauser Boston Inc., Boston, Mass, USA, 2004. 


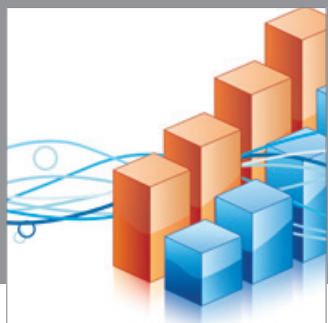

Advances in

Operations Research

mansans

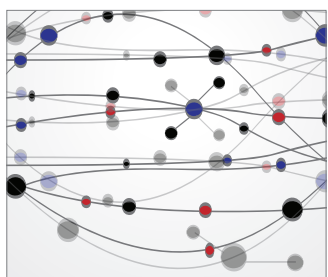

The Scientific World Journal
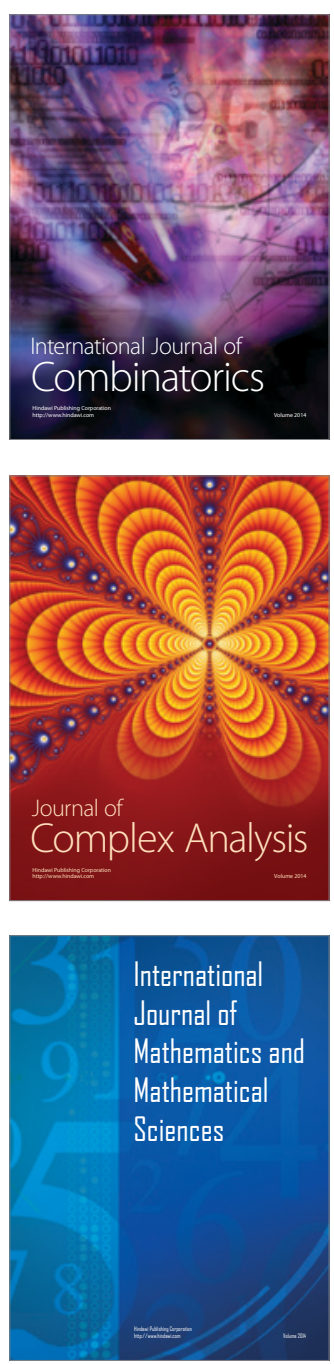
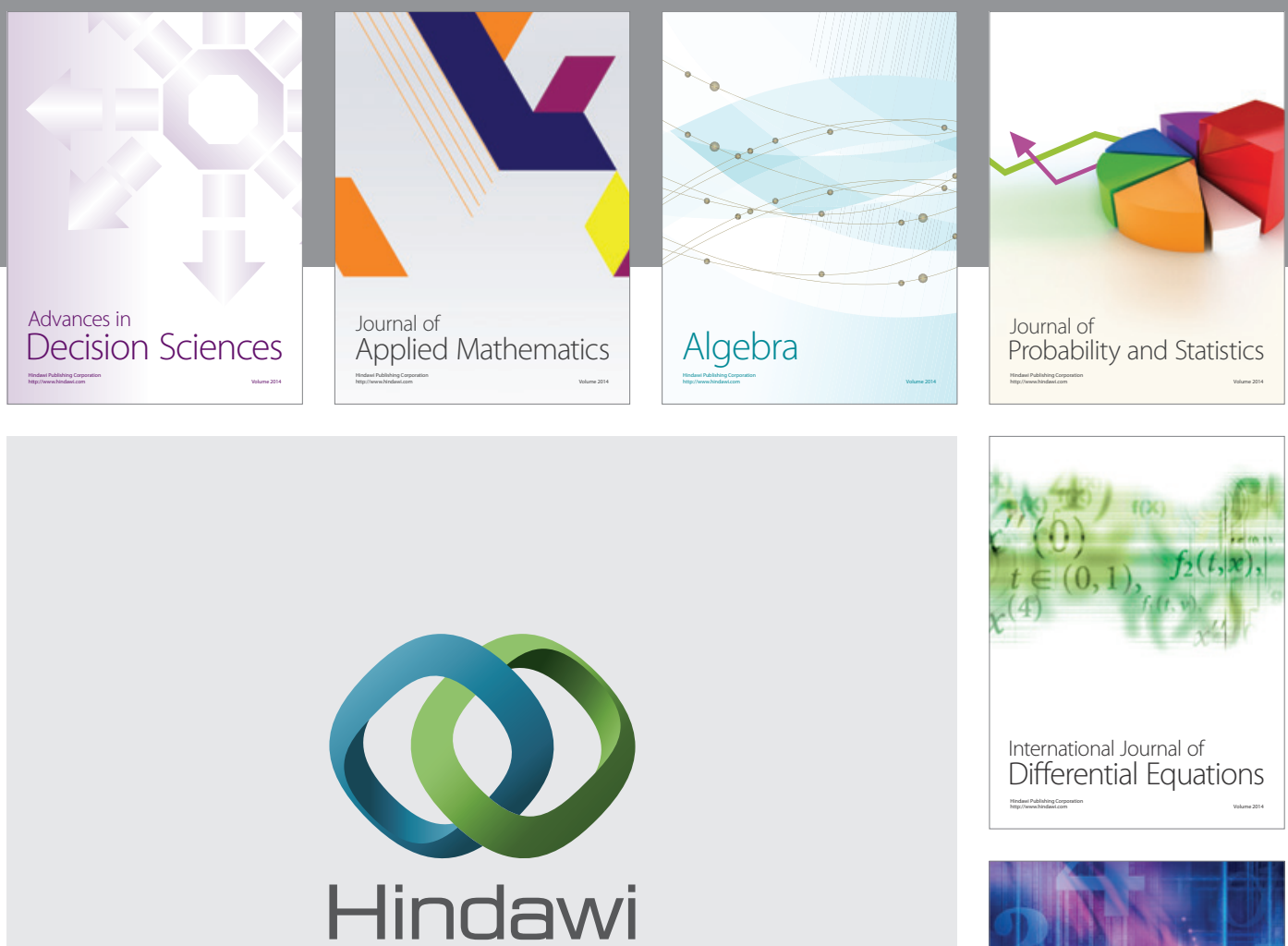

Submit your manuscripts at http://www.hindawi.com
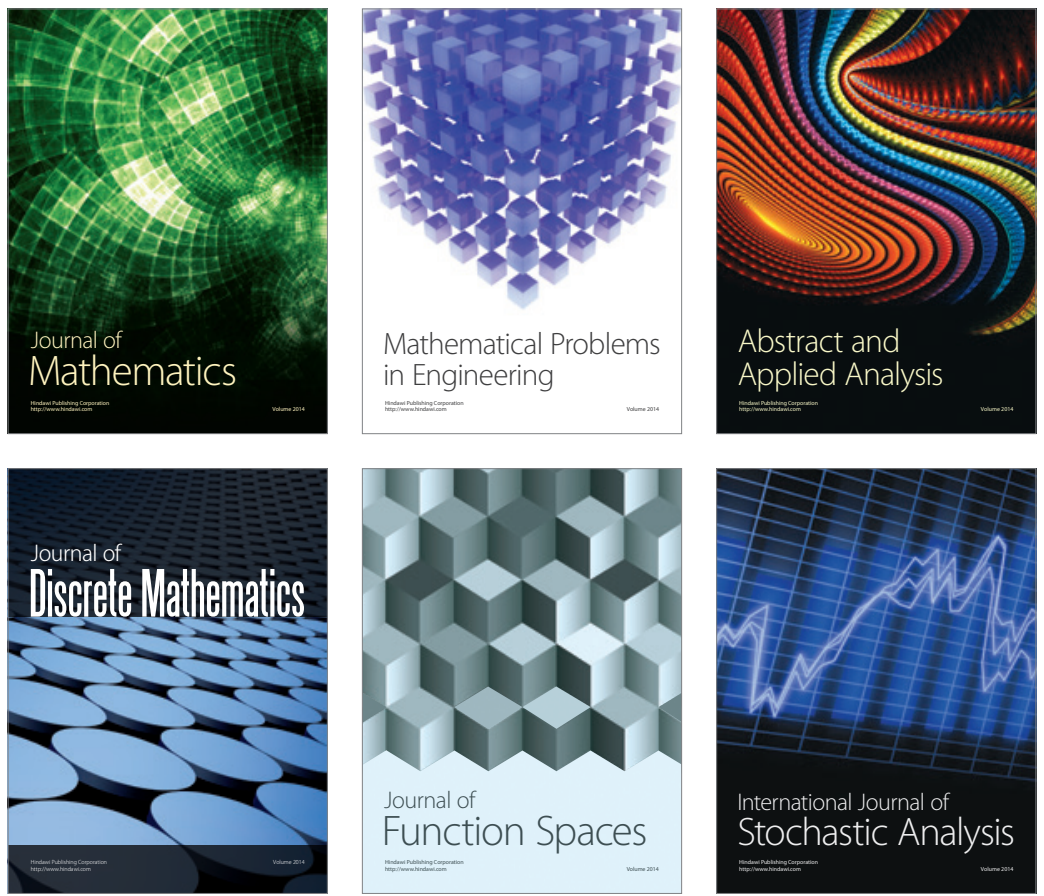

Journal of

Function Spaces

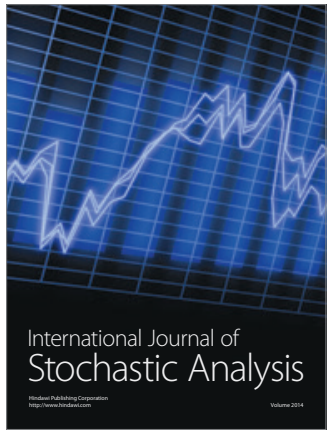

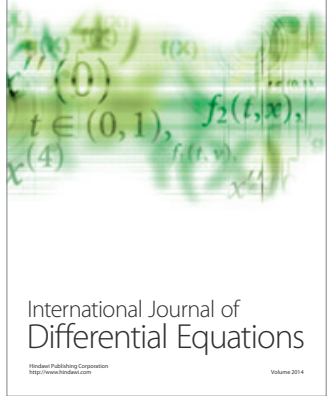
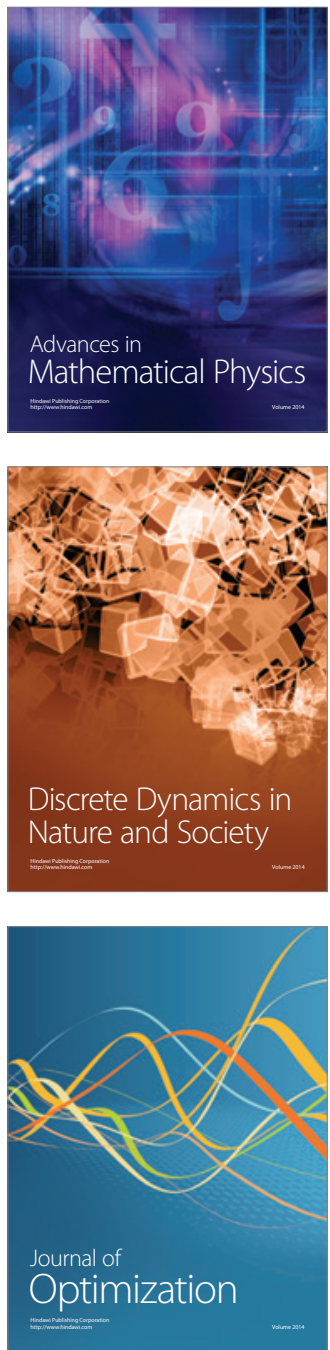